\title{
Examination and clinical correlation of olfactory system disorders by an objective method Sniffin' Sticks odor test in Parkinson's disease
}

\author{
Nur Türkmen $\odot$, Yeşim Sücüllü Karadağ๑, Zeynep Neşe Öztekin॰
}

Department of Neurology, University of Health Sciences, Ankara Numune Training and Research Hospital, Ankara, Turkey

\section{ABSTRACT}

Objectives: This study is aimed to investigate the frequency of olfactory dysfunction in Parkinson's Disease (PD) and its relationship with motor/non-motor symptoms and treatment in comparison to isolated olfactory dysfunction patients and healthy controls.

Methods: This study includes 40 PD patients, 37 anosmia patients and 42 healthy controls. PD patients are evaluated with PD evaluation form including; sociodemographic features, disease history, Unified Parkinson's Disease Rating Scale (UPDRS) score, and Hoehn and Yahr (H-Y) score. All patients were evaluated with cranial CT and MRI. Olfactory function was evaluated with Sniffin' Sticks Test (SST). A $p$ value $<0.05$ was considered to be statistically significant.

Results: The mean age and median disease duration of PD patients were $62.2 \pm 11.9$ and 4.5 years, respectively. Fifteen of them had comorbid diseases. Median UPDRS score was 19.5 (4-60) and 67.5\% of subjects were HY Stage-1. Most frequent non-motor symptom was constipation (67.5\%). Olfactory dysfunction was found in $75 \%$ of PD patients by SST. No difference was observed between PD patients with or without olfactory dysfunction regarding non-motor symptoms and dementia $(p>0.05)$. Patients with isolated olfactory dysfunction were significantly younger than both patients with PD and the healthy controls $(p<0.001)$. Nonmotor symptoms were not significantly different between isolated olfactory dysfunction group and healthy subjects $(p>0.05)$.

Conclusions: Most of the patients with PD had olfactory dysfunction, which was found not to be correlated with disease duration and stage based on the results of an objective test, namely Sniffin-Sticks odor test.This result might support the role of non-dopaminergic pathways in the etiopathogenesis of olfactory dysfunctions in PD. In clinical practice, data from further studies is required to comment on an ideal screening or diagnostic test in the olfactory system evaluation of early stage PD patients that would be repeatable and objective.

Keywords: Parkinson's disease, olfactory dysfunction, Sniffin' Sticks test, anosmia

P arkinson's disease (PD) is a progressive neurological disorder characterized by tremor, rigidity, and slowness of movements. With a lifetime risk of developing the disease of $1.5 \%, \mathrm{PD}$ is the second most prevalent neurodegenerative disorder $[1,2]$. PD is associated with progressive neuronal loss of the sub- stantia nigra and other brain structures $[1,3]$. Very few of the cases are related to mutations in; a-synuclein, leucine-rich repeat kinase- 2 and glucocerebrosidase, but the most common form is still idiopathic [3].

Although the motor symptoms of PD are well defined, the non-motor features as altered smell, taste, 
vision, cardiovascular function, sleep, gastric and bowel function, salivation, sebaceous gland activity, mood, and cognition are under-recognized and ultimately undertreated [3. 4]. Non-motor symptoms can be present at all stages of disease but the frequency generally increases with progression of disease and some studies have shown that these symptoms have a major impact on the quality of life in advance of motor symptoms $[5,6]$.

The most remarkable non-motor feature of PD is impairment in smelling with estimates of prevalence ranging from $50 \%$ to $90 \%$ [7-9]. Olfactory dysfunction is one of the earliest manifestations of PD and although definitive neural mechanisms are still unclear, they appear a few years prior to characteristic motor symptoms. While cardinal motor symptoms are primarily caused by dopamine depletion within the nigrostriatal pathway, olfactory symptoms appear to be unaffected by dopaminergic therapy, suggesting the involvement of other neurotransmitter systems and / or extranuclear pathology. A few recent studies show brain atrophy in regions related to primary olfactory and orbitofrontal cortex in early-stage PD [10, 11].

Based on literature knowledge mentioned above, this study is aimed to investigate the frequency of olfactory dysfunction in PD and its relationship with motor/non-motor symptoms and treatment in comparison with isolated olfactory dysfunction patients and healthy controls.

\section{METHODS}

In this study, it was aimed to quantitatively evaluate the olfactory disturbance of the patients who were followed up with diagnosis of Idiopathic Parkinson's Disease (IPD) at Ankara Numune Training and Research Hospital Neurology Clinic by quantifying Sniffin' Sticks odor test and to investigate the relationship between olfactory dysfunction and motor / non-motor symptoms.

The study population is consisted of 40 IPD patients who admitted to Ankara Numune Training and Research Hospital Neurology Clinic, 37 anosmia patients from Ear, Nose and Throat (ENT) Clinic and 42 healthy controls.

Inclusion criteria were (1) having a diagnosis of PD, (2) admission to the hospital with the complaint of selective olfactory sensation loss, (3) absence of any neurological diagnosis other than PD, or excluded the cases with secondary parkinsonism.

Exclusion criteria were, (1) symptoms of non-PD parkinsonism, (2) use of any drugs that cause parkinsonism, (3) to be younger than 18 years of age and (4) to avoid participation in the study.

For the control group 42 healthy volunteer, who had no age-related illness, no parkinsonism, no drug use, no sense of smell loss, no cognitive impairment to prevent cognitive co-operation were selected.

Each patient was assigned to a standard Parkinson's disease assessment form which included; sociodemographic characteristics (age, sex, occupation, education level, socioeconomic level etc.), PD and a family history of olfactory disorder, hand dominance information, disease duration and age at onset, first symptom and localization of the disease, chronological order of symptoms, Unified Parkinson's Disease Rating Scale (UPDRS) score, Hoehn and Yahr (HY) staging and information of drugs used by patients. Other systemic diseases, smoking status and head trauma history of the patients were also recorded.

In addition, presence of non-motor symptoms other than olfactory disturbance were recorded. These symptoms were assessed according to the patient's history. Patients' ear nose and throat examinations were performed at the ENT clinic and the presence of pathological findings were recorded. All patients were evaluated with cranial CT or cranial MRI.

All the patients were evaluated and tested by the same neurologist. Mini Mental State Assessment Scale was used for objective evaluation of the cognitive status of the patients. A Mini-mental status assessment score of 24 and below were considered significant in terms of dementia [12].

The Sniffin' Sticks odor test was given to the patient and control groups to quantitatively assess the sense of smell. This test consists of 12 felt-tipped pencils with different odors and the smell is released when the cover is opened. Multiple choice testing is based on the identification of daily smells. The patient chooses the one that best describes the smell from among the 4 types shown to him. Patients can choose an option to say they do not smell any odor or that they cannot identify the smell. The cut-off value for the test was set at $<7$ to be indicative of odor loss [13]. 


\section{Statistical Analysis}

Statistical analyses were performed using the SPSS for Windows software version 11.5. The variables were investigated using Kolmogorov Smirnov test to assess whether or not they are normally distributed and homogeneity of variances were asessed with Levene test. Descriptive analyses were presented as mean \pm standard deviation or median (minimum-maximum) for numerical variables, and categorical variables were reported as number of cases (n) and percentages $(\%)$. Categorical variables were assessed by Pearson's Chi-Square, Fisher's exact or Likelihood Ratio test. Whether or not there was statistically significant correlation between disease duration, UPDRS and Hoehn-Yahr stage and smell-end result in Parkinson's disease was investigated using Spearman's
Correlation test. Comparison between two groups was done with Student's t test/Mann Whitney U test and multiple groups with One-Way ANOVA/ Kruskal Wallis test. One-way ANOVA/ Kruskal Wallis tests were followed by Post Hoc Test (Tukey HSD or Conover's nonparametric multiple comparison test). A $p$ - value less than 0.05 was considered to show a statistically significant result.

\section{RESULTS}

\section{PD Features}

The mean age of $40 \mathrm{PD}$ patients were $62.2 \pm 11.9$ years, median disease duration were 4.5 years (range between 1-20 years) and 57.5\% were male. Almost all

Table 1. Basic demographic and clinical characteristics of PD patients according to olfactory function

\begin{tabular}{|c|c|c|c|}
\hline & $\begin{array}{l}\text { Normal } \\
(\mathrm{n}=10)\end{array}$ & $\begin{array}{l}\text { Olfactory dysfunction } \\
(\mathbf{n}=\mathbf{3 0})\end{array}$ & $p$-value \\
\hline Age (years) & $58.6 \pm 13.1$ & $63.4 \pm 11.4$ & $0.270 \dagger$ \\
\hline Sex & & & $0.717 \ddagger$ \\
\hline Male & $5(50.0 \%)$ & $18(60.0 \%)$ & \\
\hline Female & $5(50.0 \%)$ & $12(40.0 \%)$ & \\
\hline Smoking & $4(40.0 \%)$ & $12(40.0 \%)$ & $1.000 \%$ \\
\hline Family history & $2(20.0 \%)$ & $4(13.3 \%)$ & $0.629 \ddagger$ \\
\hline Disease Duration (years) & $10.5(1-20)$ & $3(1-15)$ & 0.0249 \\
\hline Leading symptom & & & $0.716 \ddagger$ \\
\hline Bradykinesia & $6(60.0 \%)$ & $14(48.3 \%)$ & \\
\hline Tremor & $4(40.0 \%)$ & $15(51.7 \%)$ & \\
\hline Nausea/vomiting & $3(30.0 \%)$ & $7(23.3 \%)$ & $0.689 \ddagger$ \\
\hline Postural hypotension & $5(50.0 \%)$ & $15(50.0 \%)$ & $1.000 \$$ \\
\hline Visual hallucinations & $0(0.0 \%)$ & $8(26.7 \%)$ & $0.165 t$ \\
\hline Wearing off & $1(10.0)$ & $4(13.3 \%)$ & $1.000 \ddagger$ \\
\hline On/off & $1(10.0 \%)$ & $4(13.3 \%)$ & $1.000 \%$ \\
\hline Dyskinesia & $3(30.0 \%)$ & $2(6.7 \%)$ & $0.089 \ddagger$ \\
\hline UPDRS & $23(8-60)$ & $18(4-59)$ & 0.3159 \\
\hline Hoehn-yahr & & & 0.9629 \\
\hline Stage 1 & $7(70.0 \%)$ & $20(69.0 \%)$ & \\
\hline Stage 2 & $1(10.0 \%)$ & $4(13.8 \%)$ & \\
\hline Stage 3 & $1(10.0 \%)$ & $4(13.8 \%)$ & \\
\hline Stage 4 & $1(10.0 \%)$ & $1(3.4 \%)$ & \\
\hline
\end{tabular}

$\dagger$ Student's t test, $\ddagger$ Fisher's exact test, $\uparrow$ Mann Whitney U test, \$Pearson's Chi-Square test 
the patients were right handed, $37.5 \%$ had at least one comorbid diseases $(32.5 \%$ hypotension, $20.0 \%$ diabetes mellitus, $10.0 \%$ goiter) and $15,0 \%$ had a family history of PD. Median UPDRS score of PD patients were 19.5 (range between 4-60) and 67.5\% were HY stage 1, $12.5 \%$ were HY stage 2, 12.5\% were HY stage 3 and $5.0 \%$ were HY stage 4 .

First symptom of PD were as follows; $27.5 \%$ left hand tremor, $12.5 \%$ gait disturbance, $10.0 \%$ bradykinesia, $7.5 \%$ right hand tremor. Distribution of symptoms added to PD were; $45.0 \%$ bradykinesia, $12.5 \%$ tremor, $10.0 \%$ left sided tremor and $5.0 \%$ falls and bradykinesia. Most common examination findings were; $12.5 \%$ bradykinesia, $12.5 \%$ tremor, $10.0 \%$ bradykinesia and bilateral tremor, 10.0\% bradykinesia and hypomimia. Motor and non-motor symptoms were; $50.0 \%$ bradykinesia, $47.5 \%$ tremor, $25.0 \%$ nausea and vomiting, $50.0 \%$ postural hypotension, $20.0 \%$ visual hallucinations, $12.5 \%$ wearing off, $12.5 \%$ on/off and $12.5 \%$ dyskinesia. Treatment given to patients were; 45.0 L-dopa/Benserazide, 40.0 pramipexole, 37.5 rasajilin and 35.0 L-dopa/Carbidopa/Entacapone combination.

\section{Sniffin' Sticks Odor Test}

With the olfactory system examination, 10 (47.5\%) patients had positive results but $30(75.0 \%)$ patients had positive test results according to Sniffin' Sticks odor test.

Sniffin' Sticks odor test scores of PD and isolated anosmia patients were significantly higher than those of the healthy controls $(p<0.001)$ but similar results were obtained for PD and isolated anosmia patients $(p$ $=0.21$ ).

There was no positive correlation between disease duration $(\mathrm{r}=0.28, p=0.07)$, UPDRS score $(\mathrm{r}=0.08, p$ $=0.61)$, HY stage $(\mathrm{r}=0.02, p=0.89)$ and level of odor test within the PD group. (Table 1).

\section{Comparison Between PD Patients with and with- out Olfactory Dysfunction}

There was no statistically significant difference between the groups with or without olfactory dysfunction in terms of age, gender, smoking and family history of Parkinson disease, motor symptoms, nausea and vomiting, postural hypotension, visual hallucinations, wearing off, on/off and dyskinesia, but median

Table 2. Comparison between non-motor symptoms of PD patients according to smoking

\begin{tabular}{lccc}
\hline & $\begin{array}{c}\text { No smoking } \\
(\mathbf{n}=\mathbf{2 4})\end{array}$ & $\begin{array}{c}\text { Smoking } \\
(\mathbf{n}=\mathbf{1 6})\end{array}$ & $\boldsymbol{p}$-value \\
\hline Nausea & $5(20.8 \%)$ & $6(37.5 \%)$ & $0.295 \dagger$ \\
\hline Constipation & $17(70.8 \%)$ & $10(62.5 \%)$ & $0.581 \ddagger$ \\
\hline Sialorrhea & $8(33.3 \%)$ & $6(37.5 \%)$ & $0.787 \ddagger$ \\
\hline Orthostatic & $12(50.0 \%)$ & $8(0.0 \%)$ & $1.000 \ddagger$ \\
\hline Urogenital & $8(33.3 \%)$ & $8(50.0 \%)$ & $0.292 \ddagger$ \\
\hline Incontinence & $6(25.0 \%)$ & $7(43.8 \%)$ & $0.215 \ddagger$ \\
\hline Sexual & $7(29.2 \%)$ & $8(50.0 \%)$ & $0.182 \ddagger$ \\
\hline Depression & $7(29.2 \%)$ & $5(31.3 \%)$ & $1.000 \dagger$ \\
\hline Confusion & $3(12.5 \%)$ & $1(6.3 \%)$ & $0.638 \dagger$ \\
\hline Dementia & $4(16.7 \%)$ & $2(12.5 \%)$ & $1.000 \dagger$ \\
\hline Psychosis & $1(4.2 \%)$ & $0(0.0 \%)$ & $1.000 \dagger$ \\
Sleep arrest & $7(29.2 \%)$ & $7(43.8 \%)$ & $0.343 \ddagger$ \\
\hline REM & $1(4.2 \%)$ & $1(6.3 \%)$ & $1.000 \dagger$ \\
\hline Restless Leg & $2(8.3 \%)$ & $0(0.0 \%)$ & $0.508 \dagger$ \\
\hline Periodic leg & $1(4.2 \%)$ & $0(0.0 \%)$ & $1.000 \dagger$ \\
\hline Olfactory abnormality & $13(54.2 \%)$ & $6(37.5 \%)$ & $0.301 \ddagger$ \\
\hline Abnormal Sensation & $3(12.5 \%)$ & $7(43.8 \%)$ & $0.059 \dagger$ \\
\hline
\end{tabular}

$\dagger$ Fisher's exact test, †Pearson’s Chi-square test 
disease duration was significantly lower in PD patients with olfactory dysfunction $(p=0.024)$. PD patients were also evaluated by their smoking status and no statistically significant difference was found between motor and non-motor symptoms and smoking status (Table 2). There was also no difference between olfactory dysfunction in PD patients, according to taking dopaminergic treatment or not ( $p=$ 0.604).

Comparisons Between Isolated Anosmia and Control Group

Anosmia patients' mean age was lower than PD and control groups $(p<0.001)$. Groups were similar with regard to sex and hand dominance $(p>0.05)$ (Table 3).

Mean Body Mass Index (BMI) values were simi- lar within groups. Median MMT score was significantly lower in the PD group $(p=0.03)$. There were no statistically significant differences with regard to smoking, head trauma history and PD family history between groups $(p>0.05)$. A family history of anosmia was more frequent within the anosmia group than healthy controls $(p<0.001)$. Abnormal brain MRI's were significantly less frequent within anosmia group than $\mathrm{PD}(p=0.03)$. There was statistically significant difference between groups regarding abnormal findings at ear $(p=0.03)$, head/neck $(p<0.001)$ examinations (Table 4).

With regard to non-motor features; constipation, sialorrhea, orthostatic hypotension, urogenital and sexual symptoms were more frequent in PD patients group than isolated anosmia patients and healthy control group $(p<0.05)$ (Table 5). Groups were similar

Table 3. Demographic characteristics of groups

\begin{tabular}{|c|c|c|c|c|}
\hline & $\begin{array}{l}\text { Control } \\
(n=42) \\
\end{array}$ & $\begin{array}{c}\text { Anosmia } \\
(n=37)\end{array}$ & $\begin{array}{c}\text { PD } \\
(n=40)\end{array}$ & $p$-value \\
\hline Age (years) & $61.8 \pm 10.3$ & $50.3 \pm 16.4$ & $62.2 \pm 11.9$ & $<0.001$ \\
\hline Sex & & & & 0.068 \\
\hline Male & $22(52.4 \%)$ & $12(32.4 \%)$ & $23(57.5 \%)$ & \\
\hline Female & $20(47.6 \%)$ & $25(67.6 \%)$ & $17(42.5 \%)$ & \\
\hline Hand dominance & & & & 0.526 \\
\hline Right & $40(95.2 \%)$ & $34(91.9 \%)$ & $39(97.5 \%)$ & \\
\hline Left & $2(4.8 \%)$ & $3(8.1 \%)$ & $1(2.5 \%)$ & \\
\hline
\end{tabular}

Table 4. Basic clinical characteristics of cases according to groups

\begin{tabular}{lcccc}
\hline & $\begin{array}{c}\text { Control } \\
(\mathbf{n = 4 2})\end{array}$ & $\begin{array}{c}\text { Anosmia } \\
(\mathbf{n = 3 7 )}\end{array}$ & $\begin{array}{c}\text { PD } \\
(\mathbf{n = 4 0 )}\end{array}$ & $\boldsymbol{p}$-value \\
\hline BMI $\left(\mathrm{kg} / \mathrm{m}^{2}\right)$ & $27.6 \pm 4.4$ & $27.8 \pm 4.5$ & $28.0 \pm 4.7$ & 0.933 \\
Mini mental test & $27(10-30)$ & $27(18-30)$ & $26(15-29)$ & 39 \\
Smoking & $20(47.6 \%)$ & $13(35.1 \%)$ & $16(40.0 \%)$ & 0.522 \\
Head trauma & $5(11.9 \%)$ & $6(16.2 \%)$ & $4(10.0 \%)$ & 0.704 \\
\hline PD family history & $1(2.4 \%)$ & $2(5.4 \%)$ & $6(15.0 \%)$ & 0.084 \\
Abnormal neurological finding & $2(4.8 \%)$ & - & $39(97.5 \%)$ & $<0.001$ \\
\hline Family history of anosmia & - & $7(18.9 \%)$ & $4(10.0 \%)$ & 3 \\
Abnormality in brain MRI & $18(54.5 \%)$ & $9(40.9 \%)$ & $26(74.3 \%)$ & 36 \\
\hline Head and neck problem & $14(33.3 \%)$ & $27(73.0 \%)$ & $26(65.0 \%)$ & $<0.001$ \\
Ear problem & $1(2.4 \%)$ & $4(10.8 \%)$ & - & 35 \\
Throat problem & - & - & $1(2.5 \%)$ & 0.414 \\
\hline
\end{tabular}


Table 5. Distribution of non-motor symptoms within groups

\begin{tabular}{|c|c|c|c|c|}
\hline & $\begin{array}{l}\text { Control } \\
(n=42)\end{array}$ & $\begin{array}{c}\text { Anosmia } \\
(n=37)\end{array}$ & $\begin{array}{c}\text { PD } \\
(n=40)\end{array}$ & $p$-value \\
\hline \multicolumn{5}{|l|}{ Non-motor findings } \\
\hline Nausea & $1(2.4 \%)$ & $4(10.8 \%)$ & $11(27.5 \%)$ & 0.003 \\
\hline Constipation & $8(19.0 \%)$ & $8(21.6 \%)$ & $27(67.5 \%)$ & $<0.001$ \\
\hline Sialorrhea & - & $1(2.7 \%)$ & $14(35.0 \%)$ & $<0.001$ \\
\hline \multicolumn{5}{|l|}{ Autonomic dysfunction } \\
\hline Orthostatic & $4(9.5 \%)$ & $4(10.8 \%)$ & $20(50.0 \%)$ & $<0.001$ \\
\hline Urogenital & $3(7.1 \%)$ & $5(13.5 \%)$ & $16(40.0 \%)$ & $<0.001$ \\
\hline Urinary incontinence & $9(21.4 \%)$ & $4(10.8 \%)$ & $13(32.5 \%)$ & 0.071 \\
\hline Sexual & - & $1(2.7 \%)$ & $15(37.5 \%)$ & $<0.001$ \\
\hline \multicolumn{5}{|l|}{ Psychiatric } \\
\hline Depression & $5(11.9 \%)$ & $10(27.0 \%)$ & $12(30.0 \%)$ & 0.111 \\
\hline Confusion & $2(4.8 \%)$ & $1(2.7 \%)$ & $4(10.0 \%)$ & 0.375 \\
\hline Dementia & $1(2.4 \%)$ & - & $6(15.0 \%)$ & 0.007 \\
\hline Psychosis & - & - & $1(2.5 \%)$ & 0.333 \\
\hline \multicolumn{5}{|l|}{ Sleep problems } \\
\hline Sleep disruption & $4(9.5 \%)$ & $2(5.4 \%)$ & $14(35.0 \%)$ & $<0.001$ \\
\hline REM Behaviour Disorder & - & $1(2.7 \%)$ & $2(5.0 \%)$ & 0.231 \\
\hline Restless legs & - & - & $2(5.0 \%)$ & 0.109 \\
\hline Periodic Leg Movement & - & - & $1(2.5 \%)$ & 0.333 \\
\hline \multicolumn{5}{|l|}{ Sensory findings } \\
\hline Olfactory deficit & - & $37(100.0 \%)$ & $19(47.5 \%)$ & $<0.001$ \\
\hline Other abnormal sensory findings & - & - & $10(25.0 \%)$ & $<0.001$ \\
\hline
\end{tabular}

with regard to urinary incontinence, depression, confusion, psychosis $(p<0.05)$ (Table-5).

Sniffin' Sticks odor test scores were statistically significantly higher in anosmia and PD patients than controls (both $p<0.001$ ) but results were similar at PD and anosmia patients $(p=0.21)$ (Fig. 1)

\section{DISCUSSION}

Olfactory dysfunction is an important nonmotor symptom of IPD that begins before the prodromal phase and before the appearance of characteristic motor symptoms and its pathogenesis is still not clear [14]. In this study, the odor disorder - which is known to be one of the early stage non-motor findings of the Parkinson's Disease - was investigated in relation to other features of the disease. An important objective in the handling of cases is to describe the fact that olfactory dysfunction is an unidentified etiopathogenesis in the early stage of the disease and that it is related to the other components of the disease to elaborate findings present in the approach to PD. In this study Sniffin' Sticks odor test is used to evaluate quantitative differences between groups and 30 (75.0\%) patients had positive test results, but test results were similar at PD and anosmia patients $(p=0.21)$.

An objective assessment of olfactory disturbance is a valuable method of allowing patients to obtain scientific and descriptive records without being limited to complaints about olfactory impairment. Therefore, it has been found that qualitative investigation of olfactory deficit has no place in patients' diagnosis of Parkinson and non-Parkinsonian odor disorders [15]. 


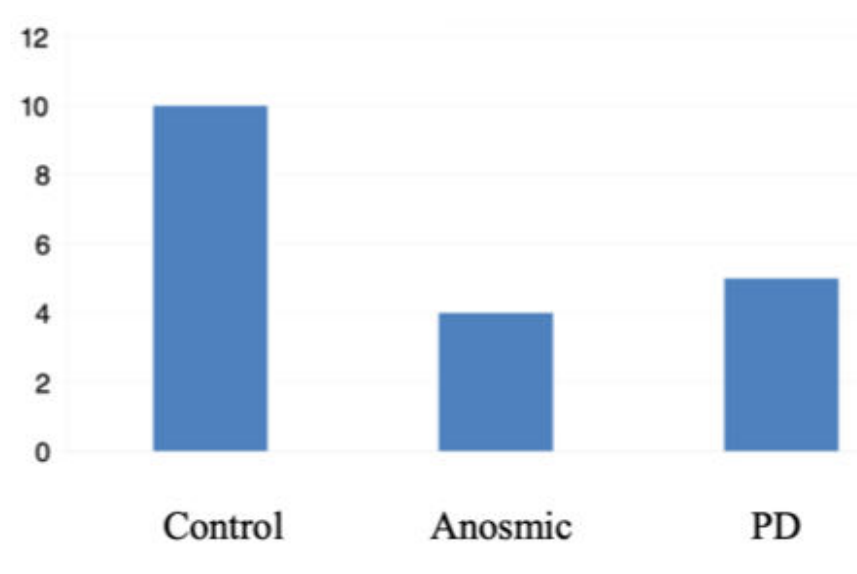

Fig. 1. Sniffin Stick odor test scores.

PD patients with impaired olfactory function had a shorter disease duration but there was no difference between the groups in terms of UPDRS scores and HY staging. On the other hand, it was determined that the olfactory disturbance was not related to motor and non-motor symptoms [8]. Similar to our results, studies showed no correlation between odor impairment and progression of motor symptoms. This result suggests that the olfactory disturbance seen early in the course of Parkinson's disease is a key finding of the disease, and the etiopathogenic mechanism might be different from the known classical mechanisms. There is a need for further screening and pathological studies of this uninformed system.

Smoking and non-smoking PD patients were similar in terms of motor and non-motor symptoms. Contradictory to our work, in one study, the odor test performances of the non-smoker group were higher than both the smoker healthy controls and the smoker and non-smoker Parkinson group [16]. In this study, according to ear and neck examinations, pathological findings were higher in the isolated anosmia group than in PD patients. This difference is thought to be significant in the etiopathology of smell loss belonging to the selective odor loss group independent of the Parkinson's disease pathogenesis.

The presence of studies demonstrating that olfactory dysfunction in Parkinson's patients results from changes in the central nervous system primarily and independently of olfactory epithelium damage [17]. Dementia was more frequent in the PD group than in anosmia patients $(p=0.007)$, but there was no difference between olfactory disorder group in PD patients $(p=0.026)$.

In a study of morphometric Magnetic Resonance detection of gray matter atrophy in the right parietal cortex, also known as primer olfactory area in Parkinson's patients, it has been shown that olfactory disturbance in Parkinson's patients is associated with gray matter atrophy [18]. In our study, percentage of patients with dementia did not differ between PD patients with and without olfactory dysfunction. However, assessment of cognitive functions with test batteries more detailed and sensitive than MMSE might have allowed for a difference between groups.

\section{CONCLUSION}

In our study, the quantitative analysis of the olfactory deficits using the Sniffin-Sticks odor test further detected odor deficit in one fourth of the patients, in addition to the subjective questioning. In clinical practice, there is a need for additional data to be obtained from studies conducted based on advanced imaging methods that include more patient groups to comment on an ideal screening or diagnostic test that can be performed which is repeatable and objective in the early stages of Parkinson's disease.

\section{Ethical approval}

All procedures performed in this study were in accordance with the ethical standards of the institutional and/or national research committee and with the 1964 Helsinki declaration and its later amendments or comparable ethical standards.

\section{Informed consent}

The study was approved by the Local Ethics Committee (Ankara Numune Training and Research Hospital, Ankara, Turkey with Ref: 2014-834). Informed consent statement was provided by patients.

\section{Conflict of interest}

The authors disclosed no conflict of interest during the preparation or publication of this manuscript.

\section{Financing}

The authors disclosed that they did not receive any grant during conduction or writing of this study. 


\section{REFERENCES}

1. Tolosa E, Wenning G, Poewe W. The diagnosis of Parkinson's disease. Lancet Neurol 2006;5:75-86.

2. Ubeda-Banon I, Saiz-Sanchez D, de la Rosa-Prieto C, Martinez-Marcos A. $\alpha$-synuclein in the olfactory system in Parkinson's disease: role of neural connections on spreading pathology. Brain Struct Funct 2014;219:1513-26.

3. Chaudhuri KR, Schapira AHV. Non-motor symptoms of Parkinson's disease: dopaminergic pathophysiology and treatment. Lancet Neurol 2009;8:464-74.

4. Wu L, Mu N, Yang F, Zang J, Zheng JP. A study of the nonmotor symptoms in early Parkinson's disease with olfactory deficits. Eur Rev Med Pharmacol Sci 2016;20:3857-62.

5. Morley JF, Duda JE. Neuropsychological correlates of olfactory dysfunction in Parkinson's disease. J Neurol Sci 2011;310:228-30.

6. Muzerengi S, Contrafatto D, Chaudhuri KR. Non-motor symptoms: identification and management. Parkinsonism Relat Disord 2007;13 Suppl 3:S450-6.

7. Berendse HW, Roos DS, Raijmakers P, Doty RL. Motor and non-motor correlates of olfactory dysfunction in Parkinson's disease. J Neurol Sci 2011;310:21-4.

8. Doty RL. Olfaction in Parkinson's disease. Parkinsonism Relat Disord 2007;13 Suppl 3:S225-8.

9. Rossi M, Perez-Lloret S, Millar Vernetti P, Drucaroff L, Costanzo E, Ballesteros D, et al. Olfactory dysfunction evaluation is not affected by comorbid depression in Parkinson's disease. Mov Disord 2015;30:1275-9.

10. Doty RL. Olfaction in Parkinson's disease and related disor ders. Neurobiol Dis 2012;46:527-52.

11. Lee EY, Eslinger PJ, Du G, Kong L, Lewis MM, Huang X. Olfactory-related cortical atrophy is associated with olfactory dysfunction in Parkinson's disease. Mov Disord 2014;29:12058 .

12. Folstein MF, Folstein SE, McHugh PR. "Mini-mental state". A practical method for grading the cognitive state of patients for the clinician. J Psychiatr Res 1975;12:189-98.

13. Hummel T, Sekinger B, Wolf SR, Pauli E, Kobal G. 'Sniffin' Sticks': olfactory performance assessed by the combined testing of odor identification, odor discrimination and olfactory threshold. Chem Senses 1997;22:39-52.

14. Hoyles K, Sharma JC. Olfactory loss as a supporting feature in the diagnosis of Parkinson's disease: a pragmatic approach. J Neurol 2013;260:2951-8.

15. Hahner A, Maboshe W, Baptista RB, Storch A, Reichmann H, Hummel T. Selective hyposmia in Parkinson's disease? J Neurol 2013;260:3158-60.

16. Moccia M, Picillo M, Erro R, Vitale C, Amboni M, Palladino $\mathrm{R}$, et al. How does smoking affect olfaction in Parkinson's disease? J Neurol Sci 2014;340:215-7.

17. Witt M, Bormann K, Gudziol V, Pehlke K, Barth K, Minovi A, et al. Biopsies of olfactory epithelium in patients with Parkinson's disease. Mov Disord 2009;24:906-14.

18. Ozelius LJ, Senthil G, Saunders-Pullman R, Ohmann E, Deligtisch A, Tagliati M, et al. LRRK2 G2019S as a cause of Parkinson's disease in Ashkenazi Jews. N Engl J Med 2006;354:424-5. 